\title{
Spurious systolic hypertension in youth: what does it really mean in clinical practice? Jean-Marie Krzesinski and Annie Saint-Remy
}

\author{
Journal of Hypertension 2006, 24:999-1001 \\ Nephrology Unit, CHU Sart Tilman, University of Liège, Liège, Belgium \\ Correspondence and requests for reprints to Professor Jean-Marie Krzesinski, \\ Department of Nephrology-Hypertension, CHU Sart Tilman, University of Liege, \\ B-4000 Liège, Belgium \\ Tel: +32 (0) 436672 03; fax: +32 (0) 436672 05; \\ e-mail: jm.krzesinski@chu.ulg.ac.be
}

\section{See original paper on page 1027}

There is a concern that hypertension in young adults may be on the increase, especially when associated with obesity. One issue of specific concern is the long-term effect of an isolated increase in systolic blood pressure detected in the first decades of life.

In clinical practice, brachial blood pressure measurement remains the classical reference for identifying hypertension, but this has been challenged recently. Indeed, estimation of central aortic pressure has been shown to be a stronger predictor of coronary artery disease [1,2] and even brings new insights regarding the effect of different antihypertensive drugs, as documented in the Conduit Artery Function Evaluation (CAFE) study. CAFE is a sub-study of the large Anglo-Scandinavian Cardiac Outcomes trial (ASCO'T) in which, despite the similar control of brachial blood pressure, aortic blood pressure was significantly lower in the amlodipine-perindopril arm compared to the betablocker-diuretic arm [3,4].

In the present issue of the journal, Hulsen et al. [5] report an original and interesting work on spurious systolic hypertension (SSH) observed in $16.2 \%$ of a large population of young adult men ( $n=352$; mean age 28 years). This phenomenon, only noted in three women $(n=398)$, is defined by a high brachial systolic blood pressure but a normal aortic systolic pressure, as estimated by a noninvasive technique using the SphygmoCor device.

Spurious systolic hypertension was first described by O'Rourke et al. [6] in a small number of asymptomatic apparently healthy young men. These young men were considered by the authors to have exaggerated amplification of the arterial pressure wave travelling to the periphery.

The existence of SSH was confirmed in another study [7] where the subjects, who were also male, appeared to be exceptionally healthy, being tall, non-smokers who were active participants in sports.

By contrast, in the study by Hulsen et al. [5], the SSH group differed from the normotensive one with respect to higher weight and body mass index. However, no significant difference in height was observed. Thus, these subjects presented another kind of profile than the one previously described, but to some extent confirming another recent publication [8]. This different aspect of the anthropometrical characteristics of $\mathrm{SSH}$ in youth makes the understanding of this observation more complex. Some important questions remain unanswered after studying the work of Hulsen et al. [5], troubling previous concepts on SSH, but opening the way for further studies in the field.

\section{What might explain the difference between aortic and brachial systolic blood pressure and why is it present mainly in males?}

The difference between gender can partly be explained by women generally being shorter than men. This leads to a lesser degree of amplification and a higher augmentation index, which has been proven to be related to height and heart rate [9] Another explanation might be due to differences in wave reflections and stroke volume, with brachial blood pressure being higher in men whereas carotid pressure is almost equal [10]. The three major factors determining where the reflected wave will meet the incident wave are the pulse wave velocity (low in young individuals), the distance the wave has to go before reflection (related to body height) and the heart rate [11]. In the study published by Hulsen $e t$ al. [5], none of these parameters differed between normotensives, true hypertensives and $\mathrm{SSH}$, giving no opportunity to explain why the central aortic systolic blood pressure is lower than the brachial one in these young men. Moreover, there was no difference in smoking or physical activity habits between groups, contrary to the observations of Mahmud and Feely [7]. By contrast, pulse pressure amplification was significantly increased in SSH, suggesting high artery elasticity. In the study by Hulsen et al. [5], it might have been interesting to measure stroke volume or hyperkinetism (no echocardiography was performed). Indeed, according to McEniery et al. [8], SSH was assimilated to isolated systolic hypertension because of common characteristics, such as high stroke volume and aortic stiffness. 


\section{How accurate is the oscillometric cuff method for brachial blood pressure measurements in the young?}

The quality of blood pressure measurement is crucial and relies on brachial blood pressure measurement in standardized conditions. In the young, as in older individuals, white-coat hypertension must be excluded. Usually, frequent repeated testings, home blood pressure measurements or better ambulatory blood pressure monitoring are required to distinguish white-coat hypertension from true hypertension. If white-coat hypertension is considered as a benign condition, the cardiovascular risk is not necessarily as low as recently published [12]. There is no certainty about having identified and excluded white-coat hypertension in the study by Hulsen $e t$ al. [5], although assessment of central pressures was performed during the second visit by non-painful radial tonometry. Therefore, this point requires validation, especially because the SphygmoCor requires a calibration that is performed using cuff blood pressure, which is less accurate than intra-arterial pressure [13].

\section{How accurate is aortic systolic blood pressure measurement?}

Controversies remain about the use of the generalized transfer function to derive aortic pressure waveform (SphygmoCor). It has been demonstrated to underestimate aortic systolic blood pressure and to overestimate aortic diastolic pressure compared with invasive measurements by cardiac catheterization [14]. Nevertheless, it has been validated in large populations [15]. In the study by Hulsen $e t$ al. [5], the small error introduced should not be a real problem because the method used was the same as that employed for the whole group of individuals.

\section{How to reach the best cardiovascular risk assessment?}

Brachial diastolic blood pressure is known to be a stronger predictor of coronary events than systolic blood pressure and pulse pressure in subjects aged less than 40 years [16]. Hulsen et al. [5] note that the SSH group presented an intermediate coronary risk. Is the prognosis so benign? Direct confirmation is still lacking and certainly deserves careful long-term follow-up to support this, although diastolic blood pressure is still in the normal range, but already significantly higher compared to the normotensive males. However, measurements of aortic systolic and pulse pressure could be more effective at all ages because aortic blood pressure relates more closely to left ventricular mass or carotid intima-media thickness than brachial blood pressure [17]. Moreover, recent data have confirmed that a blood pressure measurement usually considered as still normal could be associated with an elevated risk of cardiovascular disease, especially among those individuals with high body mass index [18].

\section{What might be the characteristics of this new kind of 'pseudo hypertension'?}

It is typical of male gender with an exaggerated pressure amplification. None of the classical cardiovascular risk factors (cholesterol, smoking, plasma glucose, etc.) differed clearly between SSH and normotensive young individuals in the Dutch study. Indeed, the diagnosis of SSH in young individuals relies on a brachial blood pressure measurement and pulse wave analysis to estimate central aortic pressure.

Obviously, this diagnosis of SSH must be completed by ambulatory blood pressure monitoring, at least to avoid confusion with white-coat hypertensive subjects. Further technical developments are needed for a more accurate estimation of central aortic pressure. Officially recognized devices must be used for brachial blood pressure measurement, which will be performed according to strict conditions. On the one hand, the results of the Dutch study emphasize the need for further studies in various young populations that could clarify the subset of determinants truly associated with SSH. On the other hand, prospective studies have to be designed to ensure that SSH is a benign condition according to the cardiovascular risk and must remain untreated as generally is the case.

\section{References}

1 Chirinos JA, Zambrano JP, Chakko S, Veerani A, Schob A, Willens H, et al. Aortic pressure augmentation predicts adverse cardiovascular events in patients with established coronary artery disease. Hypertension 2005; 45:980-985.

2 Morgan T, Lauri J, Bertram D, Anderson A. Effect of different antihypertensive drug classes on central aortic pressure. Am J Hypertens 2004; 17:118-123.

3 Williams B, Lacy PS, Thom SM, Cruickshank K, Stanton A, Collier D, et al. Differential impact of blood pressure-lowering drugs on central aortic pressure and clinical outcomes. Principal results of the Conduit Artery Function Evaluation (CAFE). Circulation 2006; 113:1213-1225.

4 Dahlöf G, Sever P, Poulter N, Wedel H, Beevers D, Caulfield M, et al. Prevention of cardiovascular events with an antihypertensive regimen of amlodipine adding perindopril as required versus atenolol adding bendroflumethiazide as required, in the Anglo-Scandinavian Cardiac Outcomes Trial-Blood Pressure Lowering Arm (ASCOT-BPLA): a multicentre randomised controlled trial. Lancet 2005; 366:895-906.

5 Hulsen H, Nijdam ME, Bos WJ, Uiterwaal C, Oren A, Grobbee D, Bots M. Spurious systolic hypertension in young adults; prevalence of high brachial systolic blood pressure and low central pressure and its determinants. J Hypertens 2006; 24:1027-1032.

6 O'Rourke M, Vlachopoulos C, Graham R. Spurious systolic hypertension in youth. Vasc Med 2000; 5:141-145.

7 Mahmud A, Feely J. Spurious systolic hypertension of youth: fit young men with elastic arteries. Am J Hypertens 2003; 16:229-232.

8 McEniery C, Yasmin, Wallace S, Maki-Petaja K, McDonnell B, Sharman JE et al. Increased stroke volume and aortic stiffness contribute to isolated systolic hypertension in young adults. Hypertension 2005; 46:221-226.

9 Smulyan H, Marchais S, Pannier B, Guerin A, Safar M, London G. Influence of body height on pulsatile arterial hemodynamic data. J Am Coll Cardiol 1998; 31:1103-1109.

10 Safar M, Smulyan H. Hypertension in women. Am J Hypertens 2004; 17:82-87.

11 Pickering T. Isolated systolic hypertension in the young. J Clin Hypertens $2004 ; 6: 47-48$.

12 Niiranen TJ, Jula AM, Kantola IM, Reunanen A. Prevalence and determinants of isolated clinic hypertension in the Finnish population: the Finn-HOME study. J Hypertens 2006; 24:463-470.

13 Smulyan H, Siddiqui D, Carlson R, London G, Safar M. Clinical utility of aortic pulses and pressures calculated from applanated radial-artery pulses. Hypertension 2003; 42:150-155. 
14 Cloud C, Rajkumar C, Kooner J, Cooke J, Bulpitt C. Estimation of central aortic pressure by sphygmoCor requires intra-arterial peripheral pressures. Clin Sci 2003; 105:219-225.

15 Wilkinson I, Cockcroft J. Estimation of central aortic pressure: shedding new light or clouding the issue? Clin Sci 2004; 106:433-437.

16 Franklin S, Larson M, Shehzad A, Khan B, Wong N, Leip E, et al. Does the relation of blood pressure to coronary heart disease risk change with aging? The Framingham Heart Study. Circulation 2001; 103:1245-1249.

17 Smulyan $\mathrm{H}$, Safar M. The diastolic blood pressure in systolic hypertension. Ann Intern Med 2000; 132:233-237.

18 Kshirsagar A, Carpenter M, Bang H, Wyatt S, Colindres R. Blood pressure usually considered normal is associated with an elevated risk of cardiovascular disease. Am J Med 2006; 119:133-141. 